\title{
LA ATENCIÓN A LA DIVERSIDAD CULTURAL Y LINGÜÍSTICA EN CONTEXTOS MIGRATORIOS. UN ANÁLISIS DESDE LA EDUCACIÓN INTERCULTURAL
} ATTENTION TO CULTURAL AND LINGUISTIC DIVERSITY IN MIGRATORY CONTEXTS. AN ANALYSIS FROM INTERCULTURAL EDUCATION

\section{Alma Arcelia RAMÍREZ ÍNIIGUEZ}

\section{Resumen}

Se presenta un análisis de las prácticas interculturales que lleva a cabo el profesorado en un contexto de migración interna. Los resultados demuestran que la formación y el compromiso en ambientes multiculturales, el reconocimiento de la diversidad de códigos, la aceptación de las diferencias mediante la colaboración, así como las expectativas en el logro de los aprendizajes son aspectos clave en la atención educativa dentro de entornos cultural y lingüísticamente diversos.

\section{Palabras clave}

Educación intercultural, migración interna, diversidad cultural, práctica pedagógica.

\begin{abstract}
The present study analyses the intercultural practices that are carried out by the teaching staff in an internal migration context. The results show that the training and commitment in multicultural environments, the acknowledgment of code diversity, the acceptance of differences through collaboration, as well as the expectations of learning achievements are key aspects in the education attention among culturally and linguistically diverse environments.
\end{abstract}

\section{Keywords}

Intercultural education, internal migration, cultural diversity, pedagogical practic.

Alma Arcelia RAMíREZ ÍÑIGUEZ. Profesora e investigadora en la Facultad de Ciencias Humanas. Universidad Autónoma de Baja California, México. Correo electrónico: alma.arcelia.ramirez.iniguez@uabc.edu.mx

Recepción: 15/VII/2020

Revisión: 26/VII/2020

Aceptación: 02/VIII/2020

Publicación: 30/IX/2020 


\section{LA ATENCIÓN A LA DIVERSIDAD CULTURAL Y LINGÜÍSTICA EN CONTEXTOS MIGRATORIOS. UN ANÁLISIS DESDE LA EDUCACIÓN INTERCULTURAL}

ATTENTION TO CULTURAL AND LINGUISTIC DIVERSITY IN MIGRATORY CONTEXTS. AN ANALYSIS FROM INTERCULTURAL EDUCATION

\section{INTRODUCCIÓN}

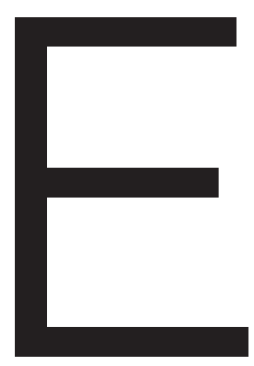

n los últimos dos años, millones de personas a nivel mundial han tenido que desplazarse por situaciones de violencia extrema en sus lugares de origen, inestabilidad económica y política, o por los efectos del cambio climático que han propiciado condiciones meteorológicas adversas, ocasionando que tengan que buscar otros entornos para vivir y desarrollarse (OIM, 2019). Específicamente, en América Latina se ha diagnosticado que las migraciones de la región hacia América del Norte han aumentado de 10 millones de personas en 1996 a 26.6 millones en 2019. Asimismo, la cantidad de personas que migran de Latinoamérica a la región europea se ha cuadriplicado desde la década de los noventa (OIM, 2019). Por su parte, la migración intrarregional se ha incrementado en los últimos años, caracterizándose por un dinamismo constante impulsado por acuerdos como el MERCOSUR, el cual ha favorecido la movilización de las poblaciones entre los países de la región y facilitado el ejercicio de los derechos laborales y de residencia (CEPAL, 2017).
De manera particular, México ha destacado como el país latinoamericano con mayor emigración, siendo el segundo país de origen de más personas migrantes en el mundo, teniendo a Estados Unidos como el principal destino (OIM, 2019). Sin embargo, también es un país de tránsito y destino de migrantes (CNDHM 2018; Higuera, 2016), especialmente para aquellos que provienen de países centroamericanos y que buscan llegar a Estados Unidos, así como de otros países de América del Sur. Además, como otros países latinoamericanos, tiene un importante flujo de migración interna. Los estudios sobre esta cuestión (Pérez y Garrido, 2019; Varela, Ocegueda y Castillo, 2017) mencionan que las políticas de promoción del empleo, basadas en el impulso al sector empresarial para el comercio internacional, han sido un factor preponderante en las causas de la migración. Al respecto, se evidencia que los efectos de dichas políticas han profundizado la desigualdad económica y social entre las distintas regiones del país. Así, las zonas con mayor desarrollo industrial han incrementado su demanda de mano de obra, destacando en las últimas décadas la industria de la agroexportación, mientras que aquellas regiones con menos inversión han empobrecido a amplios sectores 


\section{LA ATENCIÓN A LA DIVERSIDAD CULTURAL Y LINGÜÍSTICA}

Alma Arcelia RAMÍREZ İ̃̃IGUEZ

de la población como la rural, provocando que la migración sea una vía para la búsqueda del bienestar.

Los procesos migratorios contemporáneos han revelado la heterogeneidad social, económica, cultural y lingüística que ha estado presente en las sociedades, pero que los constantes flujos de personas con múltiples necesidades, características y condiciones de vida han evidenciado. Específicamente, en el campo educativo, los estudios llevados a cabo en los últimos años en los países latinoamericanos (Velasco y Rentería, 2019; Bustos y Gairín, 2017; Cigarroa, et. al., 2016; Hernández, 2016; Winikor, 2013) han demostrado que el alumnado que vive procesos de migración interna e internacional se enfrenta a problemas como la infravaloración de su cultura dentro del sistema escolar, prejuicios en relación con su desempeño académico y sus formas de vida, discriminación en las relaciones sociales o reproducción de estereotipos, o bien, obstaculización del derecho a la educación formal por estar en situación irregular o no contar con algún documento exigido por las autoridades escolares.

El contexto particular del presente estudio se refiere a la población jornalera migrante en México, quien es definida como aquellos campesinos que, por precariedad social, económica y laboral, asumen la migración como un medio de supervivencia, desplazándose periódicamente en grupos familiares completos para ofrecer su mano de obra en diversas empresas agrícolas del país, principalmente en el norte (Rojas, 2019). Al provenir de distintas localidades, el perfil cultural y lingüístico de esta población es muy diverso. Los estudios al respecto (Sánchez, Coporo y Galindo, 2020; Segura y Hernández, 2018) reconocen que una proporción importante de las familias jornaleras migrantes en México son indígenas, por lo que tienen el español como segunda lengua, o bien, no lo dominan, lo cual implica un factor relevante de diversidad cultural y lingüística en las aulas. Esta cuestión crea ambientes educativos complejos que demandan del profesorado la creación de estrategias que le posibiliten la enseñanza, tomando en cuenta la multiculturalidad y el multilingüismo. Cabe destacar que en las dinámicas educativas en las que se desarrolla la niñez de familias jornaleras migrantes se reproducen las mismas problemáticas identificadas en otros contextos migratorios de la región; es decir, viven segregación, discriminación y vulneración a su derecho a la educación.

De este modo, a pesar de que el presente estudio se centra en la revalorización de dos casos en un contexto migratorio específico, su análisis permitirá destacar la relevancia del enfoque intercultural en una de las prácticas centrales del proceso educativo como es la docente. Así, el análisis que se presenta no sólo pretende ofrecer una perspectiva dentro de un entorno particular, sino destacar la importancia de la educación intercultural en contextos de diversidad, tomando en cuenta los rasgos de la migración como uno de los procesos con mayor impacto en las sociedades en la era global. 


\section{LA ATENCIÓN A LA DIVERSIDAD CULTURAL Y LINGÜÍSTICA}

Alma Arcelia RAMÍREZ ÍNIIGUEZ

\section{EDUCACIÓN, INTERCULTURA- LIDAD Y MIGRACIÓN}

\subsection{La educación intercultural como perspectiva para atender la diversidad}

Los procesos migratorios se caracterizan por incrementar la diversidad existente en las sociedades, especialmente en lo referente a los códigos culturales y lingüísticos. Cada sociedad crea mecanismos para explicar su entorno a partir de la definición de prácticas sociales y simbólicas que establecen pautas para las relaciones sociales en las que se posibilita la formación de determinadas identidades, y en las que se suscitan múltiples dinámicas de inclusión y exclusión (Moreno y Fernández, 2017). En el caso de la institución escolar, los procesos migratorios exigen que tanto su estructura como su proyecto pedagógico respondan a las diferentes características del alumnado que, si bien era un aspecto que ya se consideraba necesario, la migración ha evidenciado y exigido la necesidad de una reconsideración de la diversidad presente en los centros educativos, especialmente la que se relaciona con la multiculturalidad y el multilingüismo. Esto último como un elemento que la migración hace explícito en los espacios escolares y que determina de manera importante el logro educativo. Así, la educación intercultural ha representado un referente teórico que ha favorecido tanto la comprensión de los procesos educativos que consideran la diversidad como uno de sus ejes principales, como el diseño de propuestas dirigidas al logro del aprendizaje de todas las personas desde sus particularidades y condiciones de vida.
En su concepción fundamental, la educación intercultural plantea como base la interacción entre los implicados en un proceso de formación, con el propósito de lograr la igualdad y la equidad en las relaciones, identificando el conflicto y las similitudes entre las distintas perspectivas tanto del alumnado como del profesorado (Valverde, 2010). Para ello, las dinámicas escolares deben partir de las características culturales del alumnado e incorporarlas en los procesos de enseñanza, de tal manera que los distintos grupos raciales, étnicos y de clase se sientan reconocidos en los ambientes escolares y experimenten la equidad educativa (Banks, 2007). Se trata de un enfoque que postula la valoración de las diferencias, asumiéndolas como una oportunidad para el enriquecimiento de los aprendizajes, especialmente de aquellos relacionados con la convivencia y la inclusión social, con la finalidad de superar la discriminación y el racismo (Morales, et. al., 2018).

En consecuencia, la educación intercultural trasciende el sentido de integración del otro a la cultura hegemónica, buscando la bidireccionalidad en los vínculos para un aprendizaje recíproco en el que se valoran los saberes y conocimientos de todos los involucrados dentro de una relación dialógica (Morales, et. al., 2018; Ávila, et. al., 2016; Escalante, Fernández y Gaete, 2014). Sin embargo, en un contexto de desigualdad económica y social como la que viven las personas que deciden emigrar como una estrategia de sobrevivencia, la perspectiva de la educación intercultural no sólo debe reconocer la existen- 
cia de diversos referentes culturales y lingüísticos sino también las estructuras que mantienen a las personas en una posición de desigualdad en relación con el entorno social en la que se insertan y que puede limitar su desarrollo.

De esta manera, la educación intercultural es un proceso que no se centra en la integración de determinados grupos a la estructura escolar ya establecida, sino que se dirige a la transformación de la propia estructura escolar, del proyecto curricular y de las prácticas de enseñanza para la inclusión de todos los referentes culturales y lingüísticos en un plano de igualdad. Es una perspectiva que modifica la visión asimilatoria de los procesos formativos para crear espacios donde el conocimiento y los aprendizajes se construyen desde las distintas identidades culturales, impulsando la participación comunitaria y los recursos disponibles en el centro escolar; siendo necesario incentivar una mirada crítica hacia los procesos de desigualdad y de exclusión simbólica que pueden replicarse en los ambientes educativos (Leiva, 2010; Mora, 2019).

Por lo tanto, la educación intercultural no se reduce a la implementación de estrategias dirigidas a la adaptación de los grupos considerados minoritarios o migrantes al sistema escolar, donde estos deben ser los interculturales, sino que se trata de una práctica en la que todos los involucrados en el proceso educativo transforman tanto su visión sobre el otro como la convivencia con aquello que les es desconocido, en ciclos de aprendizaje mutuo y continuo.

\subsection{La práctica docente desde la edu- cación intercultural}

La figura del docente adquiere un rol primordial en los procesos educativos en tanto que representa un agente socializador de los códigos culturales presentes en el ambiente escolar, por lo que puede reproducir únicamente aquellos códigos que pertenecen a la cultura hegemónica, o bien, universalizar la diversidad de referentes del alumnado migrante y local (Winikor, 2013). De esta manera, la práctica docente tiene gran relevancia en la estructura pedagógica y de gestión institucional en el que se enmarcan las acciones escolares.

Desde el marco de la educación intercultural, la práctica docente debe caracterizarse no sólo por la capacidad del profesorado para transmitir efectivamente los conocimientos, sino sobre todo por sus competencias para fomentar aprendizajes sociales a partir del reconocimiento de las diferencias y su potencial como recurso educativo para contribuir a la construcción de sociedades cada vez más democráticas y justas. En este sentido, la práctica del profesorado debe tener como eje el fomento del respeto, la tolerancia y el aprecio por las diferencias, así como el ejercicio y desarrollo de capacidades como el pensamiento crítico y la participación, la cual debe estar abierta a otros agentes educativos relevantes como las madres y padres de familia $u$ otros agentes que son parte de la comunidad y que inciden en los procesos de formación (Leiva, 2010; Valverde, 2010). Por lo tanto, más que buscar el logro de 


\section{LA ATENCIÓN A LA DIVERSIDAD CULTURAL Y LINGÜÍSTICA}

\section{Alma Arcelia RAMÍREZ ÍNIIGUEZ}

aprendizajes homogéneos en todo el alumnado, desde la perspectiva intercultural de la educación, el trabajo cotidiano del docente se centra en establecer metodologías didácticas para eliminar los prejuicios, abrir la discusión de los conflictos y fortalecer el trabajo y la comprensión mutua.

Lo anterior, demanda que el profesorado ejerza su práctica desde sus capacidades reflexivas y críticas, siendo consciente de su rol como agente de inclusión social y que, en consecuencia, desarrolle su labor hacia la construcción de una convivencia en condiciones de igualdad, asimilando su papel en la formación para una ciudadanía basada en la no discriminación (Cépeda, Castro y Lamas, 2019; Valdiviezo, 2010). Esto se traduce en la enseñanza de contenidos en los que todo el alumnado se identifique; la ampliación del repertorio de códigos de comunicación entre el alumnado, retomando su diversidad lingüística; el fomento del conocimiento de los referentes culturales de todos dentro de las actividades cotidianas en el aula; y la valoración de la cultura de cada estudiante, relacionando continuamente los contenidos escolares con los acontecimientos de la comunidad en la que todos están implicados (Cépeda, Castro y Lamas, 2019; Hamel, Erape y Márquez, 2018). Estas acciones van acompañadas de un trabajo colaborativo y horizontal con el resto del profesorado y la comunidad escolar (Ibáñez y Druker, 2018; Ramírez, 2017) a favor de una interacción educativa que contrarreste la desigualdad social en la que pueden encon- trarse los grupos considerados minoritarios y migrantes.

En síntesis, la práctica docente desde la perspectiva de la educación intercultural se caracteriza por los siguientes aspectos.

- El profesorado se asume como un agente de transformación social que, mediante su práctica, reconoce a la diversidad cultural y lingüística como parte inherente a las sociedades, comprendiendo que parte de su alumnado se encuentra en condiciones de desigualdad debido a sus condiciones de vida y a la subvaloración de sus referentes culturales y lingüísticos.

- Busca que su labor contribuya a la construcción de sociedades más justas mediante la implementación de estrategias didácticas basadas en el conocimiento y el aprendizaje mutuo, estableciendo una valoración igualitaria de todas las manifestaciones culturales y lingüísticas, preponderando aquellas que son objeto de mayor discriminación en el entorno social.

- Posee las competencias necesarias para contribuir a la resolución de conflictos originados por el racismo y la discriminación, realizando una intervención que privilegia el pensamiento crítico, la reflexión y los aprendizajes dirigidos a la convivencia social en un plano de igualdad de oportunidades y saberes.

- Realiza un trabajo colaborativo con otros agentes escolares y del entorno para otorgar sentido a los aprendizajes que se generan 
en el aula, de tal manera que se lleva a cabo una integración de los esfuerzos docentes con los que hacen otros agentes escolares y comunitarios para promover vínculos que enriquecen los aprendizajes de todas las personas.

\section{METODOLOGÍA: DOS ARQUE- TIPOS DE ESTUDIO}

La realización del presente trabajo se basó en la reinterpretación de dos casos centrados en la atención escolar de la población infantil migrante perteneciente a las familias de jornaleros agrícolas en México. Al respecto, se entiende que la construcción del conocimiento es resultado de múltiples interpretaciones y del contexto en el que estas se producen (Alarcón y Monzant, 2004), considerando que la problematización de la realidad es una acción constante que conlleva a re-articular y re-pensar las relaciones existentes en los fenómenos sociales. La reinterpretación de los casos posibilitó una nueva lectura de los significados inmersos en los procesos educativos a partir de los testimonios del profesorado, desde una perspectiva teórica actualizada de la educación intercultural, brindando lineamientos de nuevas prácticas para la atención educativa de la diversidad en contextos migratorios. Cabe señalar que este análisis permitió la comprensión de dichos significados a través del estudio de la particularidad de las circunstancias en las que suceden los hechos (Stake, 2007), destacando la excepcionalidad de cada caso (Ángel Pérez, 2011) para profundizar en la comprensión de las implicaciones de la educación intercultural desde lo local.

Dichos casos se ubican en Sinaloa, uno de los estados mexicanos que, por sus características de agroindustria de exportación ha sido uno de los principales sitios a donde llegan miles de familias jornaleras cada año. Además de contar con empresas que ofrecen servicios de vivienda, salud y alimentación, en este estado existen convenios entre las empresas y los agentes gubernamentales para asegurar la escolarización de niñas y niños, así como acciones para erradicar la explotación de mano de obra infantil. En este contexto, la reinterpretación está basada en la elección de dos escuelas con características particulares que las hacen modelos de acción dentro de este contexto. Asimismo, presentan estrategias educativas para incentivar la escolarización de la niñez migrante, teniendo en cuenta los factores de vulnerabilidad de esta población, así como prácticas docentes para atender la diversidad cultural y lingüística en las aulas. Los rasgos de los casos seleccionados son los siguientes: 


\section{LA ATENCIÓN A LA DIVERSIDAD CULTURAL Y LINGÜÍSTICA}

\section{Alma Arcelia RAMÍREZ ÍNIIGUEZ}

Tabla 1. Características de los casos de análisis.

\begin{tabular}{|c|c|c|}
\hline & CASO 1 & CASO 2 \\
\hline Tipo de escuela & $\begin{array}{l}\text { Escuela dentro de un campo agrícola } \\
\text { que dispone de servicios médicos, de } \\
\text { vivienda, alimentación, así como guar- } \\
\text { dería. La escuela sólo atiende a niñez } \\
\text { migrante a través del Programa de } \\
\text { Educación Básica para Niños y Niñas } \\
\text { de Familias Jornaleras Migrantes } \\
\text { (PRONIM). }\end{array}$ & $\begin{array}{l}\text { Escuela pública regular a la que asisten } \\
\text { niñez migrante y niñez de la localidad. } \\
\text { Se caracteriza por ser una de las prime- } \\
\text { ras escuelas públicas de la región en } \\
\text { atender a la niñez de familias jornaleras } \\
\text { migrantes. Se localiza en la comunidad } \\
\text { cercana a la empresa agrícola en la que } \\
\text { trabajan dichas familias. }\end{array}$ \\
\hline $\begin{array}{l}\text { Condiciones } \\
\text { de educabilidad }\end{array}$ & \multicolumn{2}{|c|}{$\begin{array}{l}\text { Las niñas y niños que asisten a la escuela no trabajan en el campo agrícola ni cola- } \\
\text { boran en el trabajo remunerado que realizan sus familias, por lo que se dedican a } \\
\text { las actividades escolares de tiempo completo. Asimismo, tienen sus necesidades } \\
\text { básicas cubiertas. }\end{array}$} \\
\hline Logro educativo & $\begin{array}{l}\text { El campo agrícola ha sido el primero } \\
\text { en la región en contar con servicios } \\
\text { educativos de nivel secundaria debido } \\
\text { al alto egreso del alumnado de nivel } \\
\text { primaria }\end{array}$ & $\begin{array}{l}\text { Se ha registrado mejora en el logro aca- } \\
\text { démico del alumnado, a partir de diver- } \\
\text { sas acciones emprendidas para apoyar } \\
\text { la escolarización, siendo evaluada como } \\
\text { una escuela "de calidad". }\end{array}$ \\
\hline $\begin{array}{l}\text { Apoyo educativo } \\
\text { dentro de la escuela }\end{array}$ & \multicolumn{2}{|c|}{$\begin{array}{l}\text { El profesorado tiene apoyo de un profesional en educación especial y de un psicó- } \\
\text { logo para la atención del alumnado con mayor atraso educativo. }\end{array}$} \\
\hline $\begin{array}{l}\text { Apoyo externo para } \\
\text { la escolarización }\end{array}$ & $\begin{array}{l}\text { Cuenta con servicios y una infraestruc- } \\
\text { tura que ha servido de modelo a otras } \\
\text { escuelas como área de juegos, come- } \\
\text { dor comunitario y aula de informática. }\end{array}$ & $\begin{array}{l}\text { La escuela cuenta con la infraestructura } \\
\text { suficiente para atender a la niñez que } \\
\text { llega cada ciclo agrícola a la localidad, así } \\
\text { como acuerdos con los agentes guber- } \\
\text { namentales y empresariales para brindar } \\
\text { servicios de alimentación y apoyos mate- } \\
\text { riales para la escolarización. }\end{array}$ \\
\hline
\end{tabular}

Fuente: Ramírez (2014)

El primer caso se trata de una estrategia de escolarización que ha sido prioritaria para la atención de la niñez jornalera migrante desde la década de los ochenta, la cual ha tenido el propósito de facilitar el ejercicio del derecho a la educación, adaptando los ciclos escolares a los ritmos migratorios de las familias, así como a sus características sociales y de multiculturalidad, instalando los servicios educativos dentro de los propios campos agrícolas donde viven y laboran. Sin embargo, estudios recientes al respecto (Rodríguez, Islas y Patiño, 2019; Rodríguez, 2018) señalan que los bajos resultados de esta población, a través de esta estrategia de escolarización, demandan otras alternativas educativas. Específicamente, en el estado de Sinaloa, se optó desde el año 2008 por la integración del alumnado migrante a las escuelas públicas 
más cercanas a los campos agrícolas, a donde regularmente asiste la niñez de la localidad. De acuerdo con algunos estudios (Rodríguez, Islas y Patiño, 2019), las niñas y los niños migrantes que asisten a las escuelas públicas han alcanzado mejores niveles de aprendizaje que aquellos que son atendidos por los servicios focalizados en esta población. Además, la atención desde el sistema educativo regular ha sido viable en este contexto debido a que los ciclos agrícolas son amplios, lo cual propicia que las familias se establezcan largas temporadas en los campos, facilitando la permanencia de niñas y niños en las escuelas públicas regulares, adaptando sus ciclos migratorios a los ciclos escolares. Así, la relevancia del segundo caso de análisis radica en ser la primera escuela que integró niñas y niños migrantes y desarrolló acciones educativas que han servido de guía para otras escuelas que, progresivamente, han ido escolarizando a esta población.

La recopilación de la información de los dos casos en cuestión se realizó a través de entrevistas a todo el profesorado que integra cada caso. En total, se realizaron 9 entrevistas. El tratamiento de los datos consistió en una transcripción literal de cada entrevista, con la finalidad de obtener registros de los testimonios de las y los docentes, y precisar tanto el análisis de estos como su comparación con el marco teórico de referencia. A partir de dicho registro, se llevó a cabo un análisis de contenido de la información, con base en el cual se comprendieron e interpretaron las transcripciones de las entrevistas realizadas, considerando el contexto en el que estas se produjeron (Schettini y Cortazzo, 2015). Dicho análisis se llevó a cabo a través de una codificación inductiva desde la cual se crearon las categorías que definieron los resultados del estudio (Coffey y Atkinson, 1996).

Así, los resultados destacan los siguientes aspectos sobre la atención a la diversidad cultural y lingüística desde la práctica de profesorado: 1) la formación y el compromiso en ambientes multiculturales; 2) el reconocimiento de la diversidad de códigos lingüísticos; 3) la aceptación de las diferencias culturales mediante la colaboración; y 4) las expectativas en el logro de los aprendizajes (Ramírez, 2014).

\section{RESULTADOS Y DISCUSIÓN}

\subsection{Formación y compromiso en am- bientes multiculturales}

Este aspecto de la práctica docente se evidenció en ambos casos de estudio. En relación con el primero, el profesorado expresó que la atención a la diversidad y la interculturalidad han sido parte de su formación inicial y continua. Además, algunos señalaron que su experiencia de trabajo con niñez migrante, así como la iniciativa de investigar por cuenta propia sobre el contexto de este alumnado han sido otros aspectos fundamentales que les ha ayudado a gestionar la diversidad con la que cotidianamente se encuentran en las aulas. Cabe señalar que, en el primer caso de estudio, el profesorado solamente desarrolla su práctica con niñez jornalera migrante dentro del programa PRONIM, el cual 


\section{LA ATENCIÓN A LA DIVERSIDAD CULTURAL Y LINGÜÍSTICA}

\section{Alma Arcelia RAMÍREZ Í̃̃IGUEZ}

imparte formación docente continua en la que se incluyen contenidos de educación para atender a la diversidad cultural y lingüística. A diferencia de este, en el segundo caso, el profesorado no contó con una formación específica en educación intercultural. Sin embargo, al desarrollar su práctica en una escuela pública que integra alumnado migrante y local, su experiencia le ha permitido eliminar prejuicios sobre las niñas y los niños migrantes, reconociendo sus capacidades, así como el valor de la diversidad cultural para enriquecer el aprendizaje de todo el alumnado. Por lo tanto, aunque en este último no hay una formación docente en contenidos de educación intercultural, la práctica con alumnado migrante y local le ha posibilitado al profesorado el desarrollo de competencias tanto para resolver conflictos de convivencia como para llevar a cabo un proceso de enseñanza y aprendizaje considerando la diversidad de referentes culturales presentes en el aula.

La incorporación del enfoque intercultural únicamente en la formación del profesorado del primer caso de estudio da cuenta de una perspectiva técnica-reduccionista de la educación más que una visión crítica (Leiva, 2010), a partir de la cual se concibe que la enseñanza dirigida únicamente a la niñez migrante debe considerar dicho enfoque. A pesar de lo anterior, en ambos casos de estudio, el profesorado manifiesta la necesidad de formarse en temas de interculturalidad para gestionar de manera asertiva la diversidad presente en el aula. Aunque en el primer caso, las y los docentes trabajan sólo con un sueldo sin prestaciones laborales, expresaron que trabajan con la niñez migrante por la satisfacción de contribuir a la educación de una población que se encuentra en condiciones de desigualdad social, con el propósito de coadyuvar a cambiar esta situación (Mora, 2019; Leiva, 2010). Además, en este caso, el profesorado cuenta con asesores que los apoyan en la resolución de los conflictos que se suscitan en el aula. A partir de la asesoría y los materiales de apoyo, el profesorado trabaja con otros agentes educativos para atender las distintas necesidades de aprendizaje (Ibáñez y Druker, 2018; Ramírez, 2017).

En el segundo caso, las y los docentes atienden la diversidad cultural y lingüística con la formación que de manera individual han recibido y las competencias que han desarrollado desde la experiencia en ambientes educativos con niñez migrante. Para el profesorado, esto representa un reto personal que asumen como parte de su responsabilidad y reconociendo que la atención a la diversidad es una condición indispensable para el logro académico a pesar de no contar con una formación continua en temas de interculturalidad, manifestando la necesidad de este enfoque para incluir a todo el alumnado y no reproducir los procesos de exclusión que vive la niñez migrante (Mora, 2019; Leiva, 2010).

Así, en ambos casos de estudio, las capacidades de reflexión crítica del profesorado y la conciencia de su papel en la inclusión social de la población migrante (Cépeda, Castro y Lamas, 2019; Valdiviezo, 2010) se evidencian en el compromiso y desafío que las y los docentes asumen para que el alumnado desarrolle los aprendizajes 
esperados. Esto a pesar de que el planteamiento desde el sistema educativo es que la formación en educación intercultural es sólo para el profesorado que atiende exclusivamente a la niñez migrante, con la finalidad de que esta se integre al sistema, más que la promoción de una visión dialógica, donde se considere la formación intercultural como un eje para todo el profesorado de las escuelas regulares que trabaja en ambientes multiculturales (Morales, et. al., 2018; Ávila, et. al., 2016; Escalante, Fernández y Gaete, 2014).

\subsection{Reconocimiento de la diversidad de códigos lingüísticos}

A pesar del enfoque técnico-reduccionista de la interculturalidad que se plantea desde el sistema educativo para la formación del profesorado en contextos de migración, este además de asumir un compromiso con la realidad escolar en la que se desenvuelve, desarrolla una serie de estrategias para atender la diversidad cultural y lingüística a partir de su formación, iniciativa y la experiencia que cotidianamente adquiere con su alumnado.

Entre las estrategias que emplea, en el primer caso de análisis se destacó el uso del lenguaje que utilizan niñas y niños para nombrar las cosas, así como la mención de las distintas costumbres y tradiciones para ejemplificar los contenidos que se enseñan. Además, las y los docentes propician el análisis de las ideas y opiniones que el alumnado emite sobre las manifestaciones culturales que se presentan de manera cotidiana. Asimismo, desarrollan actividades de investigación en las que el alumnado convive fuera del aula, planteando preguntas a las familias de sus compañeros, eliminando prejuicios que se puedan tener en relación con el lugar de origen de las distintas familias que llegan a vivir al campo agrícola. De este modo, se estimula la interacción del alumnado con distintos lugares de procedencia, con la finalidad de fomentar el respeto y la valoración de las diferencias culturales y lingüísticas, utilizándolas como recursos para el aprendizaje (Leiva, 2010; Valverde, 2010).

Adicionalmente, para reconocer la diversidad lingüística, llevan a cabo actividades en las que plantean las instrucciones en los distintos idiomas que se hablan en el aula con la ayuda del propio alumnado. Igualmente, realizan exposiciones y elaboran cuentos en las diferentes lenguas de las niñas y los niños. De esta manera, aunque el español es el idioma en el que el profesorado realiza su práctica docente, la diversidad lingüística es utilizada como un recurso para educar contra la discriminación y el racismo (Morales, et. al., 2018). Del mismo modo, las estrategias para reconocer dicha diversidad implican una práctica en la que se llevan a cabo aprendizajes recíprocos mediante actividades en las que el diálogo es fundamental (Morales, et. al., 2018; Escalante, Fernández y Gaete, 2014). Para ello, el ejercicio de las capacidades analíticas del profesorado es indispensable en el diseño y la evaluación de estas actividades, así como la reflexión constante de la propia experiencia, la cual es un elemento valorado como una base importante para el aprendizaje que conlleva la práctica profesional. Así, la planeación y conducción de las actividades de enseñanza contribuyen a una for- 


\section{LA ATENCIÓN A LA DIVERSIDAD CULTURAL Y LINGÜÍSTICA}

\section{Alma Arcelia RAMÍREZ ÍÑIGUEZ}

mación ciudadana basada en el reconocimiento de todos los implicados en el proceso educativo (Cépeda, Castro y Lamas, 2019; Valdiviezo, 2010).

Por su parte, en el segundo caso, el profesorado aprovecha la heterogeneidad de lugares de origen en el aula para la enseñanza de determinados contenidos como la comprensión lectora o la historia, donde propicia que el alumnado explique las características de su ciudad o pueblo de procedencia, sus costumbres y modos de vida en un ambiente en el que se comparten vivencias. Igualmente, en este caso, las y los profesores hacen uso del planteamiento de preguntas para incentivar la reflexión y el análisis de valores como la tolerancia y la aceptación, aspectos que retoman cuando se presentan conflictos o situaciones de discriminación entre compañeros. Por otro lado, consideran la diversidad lingüística cuando los contenidos del plan de estudios se relacionan directamente con dicha diversidad. Al igual que en el primer caso, se incentiva la interacción entre el alumnado, valorando las diferencias para prevenir situaciones de racismo y exclusión (Morales, et. al., 2018).

\subsection{Aceptación de las diferencias cul- turales mediante la colaboración}

El trabajo colaborativo es una estrategia que utiliza el profesorado de ambos casos de estudio tanto para propiciar el apoyo mutuo en la resolución de problemas de aprendizaje como para eliminar prejuicios entre el alumnado de diferente lugar de procedencia. También, el profesorado trabaja colaborativamente, con el propósito de profundizar en el conocimiento de las niñas y los niños que atiende, así como en la generación de ideas conjuntas para resolver los problemas de convivencia y aprendizaje. En este sentido, el vínculo que se promueve posibilita la ampliación de códigos de comunicación y la apreciación de las diferencias culturales para enriquecer los aprendizajes (Cépeda, Castro y Lamas, 2019; Hamel, Erape y Márquez, 2018), utilizando la cooperación como una estrategia que contribuye al resto de las acciones en la atención a la diversidad (Ibáñez y Druker, 2018; Ramírez, 2017).

Específicamente, en el primer caso, la comunicación con fines de colaboración entre el profesorado se extiende a otros docentes que desarrollan su práctica dentro del PRONIM en otros estados del país, logrando en algunos casos hacer un mejor seguimiento de los aprendizajes, así como conocer mejor el contexto de vida de cada niño y niña cuando este diálogo se establece con el profesorado que los ha atendido anteriormente, aunque, cabe señalar, que esta no es una actividad sistemática. En la práctica cotidiana, el trabajo colaborativo entre el profesorado sucede de manera informal en los espacios de convivencia o en las reuniones puntuales que la coordinación de PRONIM organiza, en las que tienen la oportunidad de compartir experiencias sobre su labor.

En relación con el segundo caso, entre los objetivos que tiene la cooperación entre profesores se encuentra compartir estrategias de atención 
a la diversidad, así como retroalimentar la práctica a partir de las experiencias con el alumnado que comparten. Al igual que en el primero, estas acciones se realizan de manera informal en los espacios de convivencia más que dentro de una estructura institucional que tenga como objetivo fomentar la ayuda mutua. No obstante, este aspecto sí es propiciado por el profesorado hacia las niñas y los niños, destacando que el trabajo colaborativo contribuye a la mejor comprensión de los contenidos y al conocimiento de las diversas expresiones personales y culturales en el aula, las cuales se evidencian a través de la elaboración de trabajos de manera conjunta (Morales, et. al., 2018; Ávila, et. al., 2016; Escalante, Fernández y Gaete, 2014).

En ambos casos, la migración ha propiciado que el profesorado tome la iniciativa tanto de trabajar de manera colaborativa con otros docentes, como de fomentar la cooperación y la ayuda mutua. Esto como una forma de gestionar la diversidad a través de promover la tolerancia y el respeto, impulsando el desarrollo del pensamiento crítico y la participación en las niñas y los niños, así como ejerciendo esta capacidad en su propia práctica (Leiva, 2010; Valverde, 2010).

\subsection{Las expectativas en el logro de los aprendizajes}

La experiencia y el conocimiento de las condiciones de vida de la niñez migrante, así como el ejercicio sistemático de la reflexión crítica ha provocado que el profesorado revise sus propios prejuicios en relación con las posibilidades de logro académico del alumnado que atiende. En el primer caso de análisis, las y los docentes destacan que la niñez migrante tiene una actitud positiva para el aprendizaje, mostrando interés para desarrollar las actividades escolares. Al respecto, destacan la ventaja de que las niñas y los niños que atienden no formen parte de la mano de obra para las empresas agrícolas como suele ocurrir con la niñez jornalera en todo el país, por lo que muestran más interés por el aprendizaje escolar.

Por su parte, en el segundo caso, se evidencia de manera más contundente la formación de expectativas positivas en el logro de los aprendizajes, en tanto que, al estar conviviendo la niñez migrante con la local, el profesorado observa las capacidades y la disposición para el aprendizaje del alumnado en su conjunto, distinguiendo que estos aspectos no dependen de los referentes culturales sino de una serie de factores asociados a las características individuales. En este sentido, las y los profesores mencionaron que la diversidad cultural es un punto de partida para enriquecer el desarrollo de aprendizajes académicos y sociales, y no un obstáculo en el logro educativo.

La integración de la niñez migrante a la escuela pública, además de enriquecer los referentes culturales y sociales a partir de la convivencia de alumnado con diferentes condiciones de vida, contribuye a que se disminuyan prejuicios ante las niñas y los niños en mayor vulnerabilidad educativa y social, tanto por parte del alumnado como del profesorado que desarrolla su práctica en un entorno con alta diversidad cultural y lingüística. 


\section{LA ATENCIÓN A LA DIVERSIDAD CULTURAL Y LINGÜÍSTICA}

\section{Alma Arcelia RAMÍREZ ÍÑIGUEZ}

Así, las expectativas que se forman en relación con el logro de los aprendizajes se basan en una evaluación continua, formal e informal, de las competencias que progresivamente el alumnado va logrando a partir de las distintas actividades de enseñanza y de aprendizaje (Cépeda, Castro y Lamas, 2019; Hamel, Erape y Márquez, 2018).

\section{CONCLUSIONES}

Las características de las migraciones contemporáneas demuestran que la movilidad de las personas está fuertemente determinada por las condiciones de precariedad laboral, económica y social que limitan el ejercicio pleno de sus derechos humanos y ciudadanos. Por lo tanto, su atención desde diversos ámbitos sociales e institucionales es un aspecto esencial en la mejora de sus posibilidades de bienestar. Dentro de dichos ámbitos, el educativo tiene especial relevancia al constituir un proceso mediante el cual se adquieren competencias que promueven un desarrollo que rebasan el aspecto económico, posibilitando la formación de ciudadanos participativos en la defensa y el ejercicio de sus derechos para una vida plena (Echeita y Navarro, 2014). Para ello, la atención a la diversidad cultural y lingüística que conforman los entornos educativos a partir de la integración de la población migrante resulta un elemento básico en el logro de dichas competencias.

Si bien la educación desde una perspectiva intercultural no radica exclusivamente en la práctica del profesorado, este es un elemento fundamental por su papel vehicular entre los objetivos del sistema educativo y el logro de los aprendizajes (Winikor, 2013), siendo un agente central en las prácticas sociales que incluyen o excluyen a ciertas identidades culturales (Moreno y Fernández, 2017). Los arquetipos de estudio que se presentaron en este trabajo, además de haber sido relevantes en el contexto particular en el que se ubican, destacan también por mostrar la complejidad de los factores que inciden en la formación del alumnado migrante, considerando las ventajas y limitaciones a las que se enfrenta el profesorado para atender la diversidad.

En el análisis de los casos presentados, se destaca que a pesar de que la perspectiva de educación intercultural es técnico-reduccionista (Leiva, 2010), el profesorado lleva a cabo una práctica crítica fundamentada en la comprensión de la desigualdad social de la población migrante, buscando la equidad en las relaciones educativas y sociales para evitar prácticas de exclusión (Morales, et. al., 2018; Valverde, 2010). A partir de este análisis, es posible concluir que existen diversos enfoques de educación intercultural, pero que debe prevalecer aquel que privilegia el diálogo basado en el reconocimiento de saberes, con el propósito de lograr una justicia cognitiva (Ávila, et. al., 2016) que propicie la justicia social. Por ello, la práctica docente debe orientarse al desarrollo de aprendizajes desde la reflexión, la cooperación, la participación y el trabajo diario para la transformación de los aspectos que causan exclusión, discriminación o racismo. 


\section{LA ATENCIÓN A LA DIVERSIDAD CULTURAL Y LINGÜÍSTICA}

Alma Arcelia RAMÍREZ İ̃̃IGUEZ

Con base en lo anterior, es posible sintetizar los siguientes factores como centrales en los procesos educativos que se proponen atender la diversidad cultural y lingüística en entornos de alta heterogeneidad.

- La interculturalidad debe ser la base de la formación básica y continua del profesorado, dentro de la cual se retomen tanto contenidos específicos de atención a la diversidad, como la experiencia cotidiana de las y los docentes dentro de las dinámicas de enseñanza y aprendizaje, impulsando el desarrollo de la capacidad crítica y la relevancia de su papel en la mejora de las condiciones de vida del alumnado socialmente más vulnerable.

- Dicha formación debe orientarse al desarrollo de habilidades didácticas que no se limiten a la instrucción de contenidos académicos, sino que propicien el pensamiento crítico en el alumnado para analizar las situaciones de conflicto y la comprensión de la diversidad como un aspecto implícito en el contexto tanto escolar como social.

- El contacto del profesorado con las condiciones de vida del alumnado migrante fomenta el desarrollo de un compromiso profesional para mejorar sus oportunidades de vida, por lo que la educación intercultural se convierte en una herramienta para guiar procesos educativos inclusivos basados en la implementación de estrategias que partan de los saberes y referentes del alumnado.

- El trabajo cooperativo es un factor indispensable para crear ambientes educativos inclusivos en contextos de diversidad. La colaboración entre las y los estudiantes permite que amplíen el conocimiento sobre los referentes de vida de sus colegas de clase, eliminando prejuicios y discriminación e incentivando el apoyo mutuo.

- La colaboración entre el profesorado es una estrategia efectiva para resolver problemas relacionados con la atención a la diversidad y para compartir acciones que propicien el aprendizaje de todos. En este sentido, una estructura institucional que fomente la cultura de la cooperación como parte de la vida cotidiana resulta favorable en la gestión efectiva de la diversidad, siendo el trabajo integrado una estrategia para resolver conjuntamente los retos que implica la complejidad de ambientes educativos heterogéneos.

En resumen, la perspectiva intercultural de la educación no se reduce a la atención a la diversidad sólo en el alumnado migrante o aquel que se considera culturalmente minoritario para que sea integrado al sistema escolar, sino que asume que las y los estudiantes son diversos, y que las distintas expresiones culturales y lingüísticas forman parte de los ambientes educativos, enriqueciendo el aprendizaje y buscando la transformación de las condiciones que segregan al alumnado más vulnerable a la exclusión social.

\section{REFERENCIAS BIBLIOGRÁFICAS}

Alarcón, J. y Monzant, J. L. (2004). Ciencias sociales, historia y cultura. Construcción de nuevas tendencias teóricas. Revista de Ciencias Humanas y Sociales, 20(45), 29-42. 


\section{LA ATENCIÓN A LA DIVERSIDAD CULTURAL Y LINGÜÍSTICA}

\section{Alma Arcelia RAMÍREZ ÍÑIGUEZ}

Ángel Pérez, D. (2011). La hermenéutica y los métodos de investigación en Ciencias Sociales. Estudios de Filosofía, 44, 9-37.

Ávila, L., Betancourt, A., Arias, G. y Ávila, A. (2016). Vinculación comunitaria y diálogo en la educación superior intercultural en México. Revista Mexicana de Investigación Educativa, 21(70), 759-783.

Banks, J. (2007). Educating Citizens in a Multicultural Society. Nueva York: Teachers College Press.

Bustos, R. y Gairín, J. (2017). Adaptación académica de estudiantes migrantes en contexto de frontera. Calidad en la educación, 46, 193-220. http://dx.doi.org/10.4067/ S0718-45652017000100193.

Cépeda, N., Castro, D. y Lamas, P. (2019). Concepciones de interculturalidad y práctica en aula: estudio con maestros de comunidades shipibas en el Perú. Educación, 28(54), 61-86. https://doi.org/10.18800/educacion.201901.004.

Cigarroa, R. I., Rojas, M. L., Evangelista, A. A. y Saldívar, A. (2016). Educación inclusiva y niñez migrante: barreras a la educación de niñez de origen centroamericano en escuelas de Tapachula, Chiapas (México). Revista de Investigación en Educación, 14(1), 85-104.

Comisión Económica para América Latina y el Caribe. (2017). Informe final de la reunión regional latinoamericana y caribeña de expertas y expertos en migración internacional preparatoria del pacto mundial para una migración segura, ordenada y regular. CEPAL. Coffey, A. y Atkinson, P. (1996). Encontrar sentido a los datos cualitativos. Estrategias comple- mentarias de investigación. Universidad de Antioquia.

Comisión Nacional de Derechos Humanos. (2018). Los desafíos de la migración y los albergues como oasis. Encuesta nacional de personas migrantes en tránsito por México. CNDH.

Echeita, G. y Navarro, D. (2014). Educación inclusiva y desarrollo sustentable. Una llamada urgente a pensarlas juntas. Edetania, 46, 141-161.

Escalante, C., Fernández, D. y Gaete, M. (2014). Práctica docente en contextos multiculturales: Lecciones para la formación en competencias docentes interculturales. Revista Electrónica Educare, 18(2), 71-93. http:// dx.doi.org/10.15359/ree.18-2.4.

Hamel, R. E., Erape, A. E. y Márquez, B. (2018). La construcción de la identidad purépecha a partir de la educación intercultural bilingüe propia. Trabalhos em Linguística Aplicada, 57(3), 1377-1412. https://doi.org/10.159 0/010318138653739444541.

Hernández, A. (2016). El currículo en contextos de estudiantes migrantes: Las complejidades del desarrollo curricular desde la perspectiva de docentes de aula. Estudios Pedagógicos, 42(2), 151 169. http://dx.doi.org/10.4067/S071807052016000200009.

Higuera, L.E. (2016). Fronteras polarizadas, realidades compartidas: ¿y los derechos de la población infantil en tránsito? En Moreno, J.A., Valencia, C. y L.M. Niño. (Coord.). Estudios fronterizos: nuevos escenarios de la migración (pp. 163-179). Universidad Autónoma de Baja California. 
Ibáñez, N. y Druker, S. (2018). La educación intercultural en Chile desde la perspectiva de los actores: Una co-construcción. Convergencia Revista de Ciencias Sociales, 78, 227-249. https://doi.org/10.29101/crcs. v25i78.9788.

Leiva, J. J. (2010). Educación intercultural y convivencia desde la perspectiva docente. Profesorado. Revista de currículum y formación del profesorado, 14(3), 251-274.

Mora, M. L. (2019). Aportes de la filosofía intercultural latinoamericana para la gestión de la diversidad cultural migrante en la escuela. Estudios. Utopía y praxis latinoamericana, 24, 78-85.

Morales, S., Quintriqueo, S., Uribe, P. A. y Arias, K. (2018). Interculturalidad en educación superior: experiencia en educación inicial en La Araucanía, Chile. Convergencia. Revista de Ciencias Sociales, 77, 55-76. https://doi. org/10.29101/crcs.v25i77.4706.

Moreno, K. y Fernández, Z. (2017). Educación intercultural bilingüe y relaciones interétnicas en la Escuela "Creación Yukpa" del Municipio San Francisco, Estado Zulia, Venezuela. Espacio Abierto. Cuaderno Venezolano de Sociología, 26(1), 139-155.

Organización Internacional para las Migraciones. (2019). Informe sobre las Migraciones en el Mundo 2020. OIM.

Pérez, E. y Garrido, L. A. (2019). Migración rural interna en México. Tendencias recientes: 2000-2015. En Castillo, G. (Coord.). Migraciones internas en México. Miradas desde la geografía humana (pp. 15-26). Universidad Nacional Autónoma de México.
Ramírez, A. (2017). La educación con sentido comunitario. Reflexiones en torno a la formación del profesorado. Educación, 26(51), 79-94. https://doi.org/10.18800/educacion.201702.004.

Ramírez, A. (2014). La educación escolar como factor de inclusión social desde un planteamiento sistémico. El caso de la niñez jornalera migrante en los valles de Culiacán, Sinaloa (México) (tesis doctoral). Universidad de Barcelona.

Rodríguez, C. R. (2018). Éxito escolar de estudiantes de familias de jornaleros migrantes en México. En Rodríguez, C. R. y Rojas, T. (Coords.). Migración interna, infancia y derecho a la educación. Aproximaciones interdisciplinarias, actores y propuestas de políticas públicas (pp. 153-180). Universidad Iberoamericana.

Rodríguez, C. R., Islas, J. M. y Patino, P. (2019). Rendimiento académico de los alumnos que asisten a escuelas para migrantes en México. Revista Latinoamericana de Estudios Educativos, 49(2), 59-86.

Rojas, T. (2019). Trayectorias escolares de hijos de familias agrícolas migrantes en Sonora, México. Región y Sociedad, 31, 1-21. https:// doi.org/10.22198/rys2019/31/1181.

Sánchez, M. A., Coporo, G. y Galindo, J. A. (2020). Defensoría de jornaleros agrícolas migrantes del estado de Chiapas. Cuadernos Intercambio sobre Centroamérica y el Caribe, 17(1), 1-21. https://doi.org/10.15517/c.a.. v17i1.40364.

Schettini, P. y Cortazzo, I. (2015). Análisis de datos cualitativos en la investigación social. 


\section{LA ATENCIÓN A LA DIVERSIDAD CULTURAL Y LINGÜÍSTICA}

\section{Alma Arcelia RAMÍREZ ÍÑIGUEZ}

Procedimientos y herramientas para la interpretación de información cualitativa. Universidad Nacional de la Plata.

Segura, T.y Hernández, O. (2018). Niñez inmigrante e interculturalidad escolar en el norte de Chihuahua. Región y Sociedad, 30, 1-27. http:// dx.doi.org/10.22198/rys.2018.73.a929.

Stake, R. (2007). Investigación con estudios de casos. Ediciones Morata.

Valverde, A. (2010). La formación docente para una educación intercultural en la escuela secundaria. Cuicuilco, 48, 133-147.

Valdiviezo, R. (2010). La dimensión ética en la formación docente fundada en una pedagogía de preocupación por los "otros". Revista Integra Educativa, 3(3), 139-163.
Varela, R., Oceguerda, J. M. y Castillo, R. (2017). Migración interna en México y causas de su movilidad. Perfiles Latinoamericanos, 25(49), 141-167. http://dx.doi.org/10.18504/ pl2549-007-2017.

Velasco, L. y Rentería, D. (2019). Diversidad e interculturalidad: la escuela indígena en contextos de migración. Estudios Fronterizos, 20, 1-28. https://doi.org/10.21670/ ref.1901022.

Winikor, M. (2013). De prejuicios y disciplinamientos. La mirada docente y el rol de la escuela pública frente a los inmigrantes limítrofes que llegan a la Argentina. Revista Integra Educativa, 6(1), 43-55. 